\title{
Quasilinear systems with linearizable characteristic webs
}

Cite as: J. Math. Phys. 58, 071506 (2017); https://doi.org/10.1063/1.4994198

Submitted: 09 June 2016 . Accepted: 02 July 2017 . Published Online: 20 July 2017

S. I. Agafonov, E. V. Ferapontov, and V. S. Novikov (D)
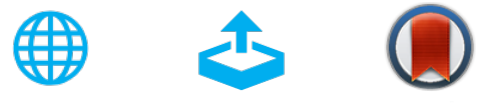

View Online

\section{ARTICLES YOU MAY BE INTERESTED IN}

Analytic properties of the electromagnetic Green's function Journal of Mathematical Physics 58, 071501 (2017); https:// doi.org/10.1063/1.4993199

The exact solution of the Schrödinger equation with a polynomially spatially varying mass

Journal of Mathematical Physics 58, 072103 (2017); https:// doi.org/10.1063/1.4993194

Global existence of weak solution for quantum Navier-Stokes-Poisson equations Journal of Mathematical Physics 58, 071507 (2017); https: //

doi.org/10.1063/1.4996015

Where in the world is AIP Publishing? Find out where we are exhibiting next 


\title{
Quasilinear systems with linearizable characteristic webs
}

\author{
S. I. Agafonov, ${ }^{1, a)}$ E. V. Ferapontov, ${ }^{2, a)}$ and V. S. Novikov, ${ }^{2, a)}$ \\ ${ }^{1}$ Departamento de Matemática, UNESP-Universidade Estadual Paulista, \\ São José do Rio Preto, Brazil \\ ${ }^{2}$ Department of Mathematical Sciences, Loughborough University, Loughborough, \\ Leicestershire LE11 3TU, United Kingdom
}

(Received 9 June 2016; accepted 2 July 2017; published online 20 July 2017)

\begin{abstract}
We classify quasilinear systems in Riemann invariants whose characteristic webs are linearizable on every solution. Although the linearizability of an individual web is a rather nontrivial differential constraint, the requirement of linearizability of characteristic webs on all solutions imposes simple second-order constraints for the characteristic speeds of the system. It is demonstrated that every such system with $n>3$ components can be transformed by a reciprocal transformation to $n$ uncoupled Hopf equations. All our considerations are local. Published by AIP Publishing. [http://dx.doi.org/10.1063/1.4994198]
\end{abstract}

\section{INTRODUCTION}

In this paper, we investigate the geometry of characteristics of quasilinear systems in Riemann invariants,

$$
R_{t}^{i}=\lambda^{i}(R) R_{x}^{i}
$$

$i=1, \ldots, n$ (no summation over $i$ ). Systems of this form govern a wide range of problems in pure and applied mathematics. Let us recall the basic concepts needed to state our main results.

Semi-Hamiltonian property. System (1) is called semi-Hamiltonian if its characteristic speeds $\lambda^{i}$ satisfy the constraints,

$$
\partial_{k}\left(\frac{\partial_{j} \lambda^{i}}{\lambda^{j}-\lambda^{i}}\right)=\partial_{j}\left(\frac{\partial_{k} \lambda^{i}}{\lambda^{k}-\lambda^{i}}\right),
$$

where $\partial_{i}=\partial / \partial R^{i}$. It was shown by Tsarev ${ }^{22}$ that this property is equivalent to integrability: semiHamiltonian systems (1) possess infinitely many conservation laws and commuting flows and can be solved by the generalised hodograph method.

Linear degeneracy. System (1) is said to be linearly degenerate if its characteristic speeds satisfy the conditions,

$$
\partial_{i} \lambda^{i}=0,
$$

no summation, $i=1, \ldots, n$. Linear degeneracy is known to prevent the breakdown of smooth initial data, which is typical for genuinely nonlinear systems of type (1), and has been thoroughly investigated in the literature, see, e.g., Refs. 16, 19, and 21.

Reciprocal transformations. There exists a natural class of reciprocal transformations acting on systems of type (1). These are non-local changes of the independent variables, $(x, t) \rightarrow(\tilde{x}, \tilde{t})$, defined as

$$
d \tilde{x}=A d t+B d x, \quad d \tilde{t}=M d t+N d x,
$$

where the right-hand sides are two conservation laws of system (1), that is, two 1-forms that are closed on every solution ( $A, B, M$, and $N$ are the functions of $R$ 's). The transformed system reads

$$
R_{\tilde{t}}^{i}=\tilde{\lambda}^{i}(R) R_{\tilde{x}}^{i},
$$

\footnotetext{
a)Electronic addresses: agafonov@ibilce.unesp.br; E.V.Ferapontov@lboro.ac.uk; and V.S.Novikov@lboro.ac.uk 
where the transformed characteristic speeds are given by

$$
\tilde{\lambda}^{i}=\frac{\lambda^{i} B-A}{M-\lambda^{i} N}
$$

Reciprocal transformations are known to preserve both the semi-Hamiltonian property and the linear degeneracy. ${ }^{8}$

Characteristics. Characteristic curves of the $i$ th family are defined by the equation $d x+\lambda^{i} d t=0$. Altogether, characteristics form an $n$-web (that is, $n$ one-parameter families of curves) on every solution. We refer to Refs. 3 and 4 for an introduction to the web geometry.

Parallelizable webs. An $n$-web is said to be parallelizable if it is locally diffeomorphic to $n$ families of parallel lines (parallelizable 3-webs are also known as hexagonal). The following result provides a link between the above concepts.

Theorem 1 (Refs. 9 and 10). The following conditions are equivalent:

(a) System (1) has a parallelizable characteristic web on every solution.

(b) System (1) can be linearized by a reciprocal transformation.

(c) System (1) satisfies the following conditions:

- semi-Hamiltonian property;

- linear degeneracy;

- every quadruple of characteristic speeds has a constant cross-ratio (for $n=3$ this condition becomes redundant).

Linearizable webs. An $n$-web is said to be linearizable (rectifiable) if it is locally diffeomorphic to $n$ families of lines, not necessarily parallel. We emphasize that the condition of linearizability is far more subtle than that of parallelizability, see Refs. $1-3,5,6,12,13$, and 15 and references therein for a discussion of the linearizability problem.

The aim of this paper is to establish an analogue of Theorem 1 for systems (1) whose characteristic webs are linearizable on every solution. Our main observation is that, although the linearizability of an individual web is a rather nontrivial differential constraint, the requirement of linearizability of characteristic webs on all solutions leads to simple second-order differential constraints for the characteristic speeds, see Theorem 3. In particular, any such system is reciprocally related to $n$ uncoupled Hopf equations, $R_{t}^{i}=f^{i}\left(R^{i}\right) R_{x}^{i}$.

\section{LINEARIZABILITY OF A PLANAR WEB}

Any projective transformation takes a linear web into a linear web. Thus, the linearizability of a web can be expressed in terms of projective differential invariants of the linearizing map, namely, its Schwarzian derivative. ${ }^{20}$ This approach can be traced back to the pioneering work of Bol. ${ }^{5,6}$ The following form of the linearizability criterion will be most convenient for our purposes:

Theorem 2 (Ref. 15). A planar n-web formed by integral curves of vector fields,

$$
\left\{V_{i}=\partial_{t}-\lambda^{i}(t, x) \partial_{x}\right\}
$$

is linearizable if and only if there exists a solution $E, F, G$, and $H$ of the following system:

$$
\begin{aligned}
& 2 G_{t x}+H_{x x}+F_{t t}-6 G G_{x}+2 H E_{t}+E H_{t}+3 F H_{x}-3 G F_{t}+3 H F_{x}=0, \\
& G_{x x}+E_{t t}+2 F_{t x}+3 E G_{t}-3 F G_{x}+3 G E_{t}+H E_{x}+2 E H_{x}-6 F F_{t}=0,
\end{aligned}
$$

subject to the constraints

$$
E\left(\lambda^{i}\right)^{3}+3 F\left(\lambda^{i}\right)^{2}+3 G \lambda^{i}+H=V_{i}\left(\lambda^{i}\right), \quad i=1, \ldots, n .
$$


Remark. The functions $E, F, G$, and $H$ are linearly independent components of the 2-dimensional Schwarzian derivative of the linearizing map. ${ }^{1}$ For $n \geq 4$, relations (5) uniquely define $E, F, G$, and $H$, and Eq. (4) leads to explicit second-order constraints for $\lambda^{i}$. On the contrary, for $n=3$, relations (5) are only sufficient to determine, say, $E, F$, and $G$ in terms of $H$, so that relations (4) give an overdetermined second-order system for $H$. The analysis of this system is quite involved; in particular, differential constraints for $\lambda^{i}$ appear at differential order six and higher. This explains why we treat the cases $n \geq 4$ and $n=3$ separately.

\section{III. $n \times n$ SYSTEMS WITH LINEARIZABLE CHARACTERISTICS $(n \geq 4)$}

The main result of this paper is the following theorem.

Theorem 3. For $n \geq 4$, the following conditions are equivalent:

(a) System (1) has a linearizable characteristic web on every solution.

(b) System (1) can be transformed by a reciprocal transformation to $n$ uncoupled Hopf equations.

(c) Characteristic speeds of system (1) satisfy the following conditions:

- For every quadruple of pairwise distinct indices $i, j, k$, and $l$, one has a relation

$$
a_{i j}\left(\lambda^{k}-\lambda^{l}\right)+a_{k j}\left(\lambda^{l}-\lambda^{i}\right)+a_{l j}\left(\lambda^{i}-\lambda^{k}\right)=0,
$$

where $a_{i j}=\frac{\partial_{j} \lambda^{i}}{\lambda^{j}-\lambda^{i}}$. This allows one to introduce the parametrization $a_{i j}=p_{j} \lambda^{i}+q_{j}$.

- The 1-forms

$$
\omega_{11}=\sum_{i=1}^{n} p_{i} \lambda^{i} d R^{i}, \quad \omega_{12}=\sum_{i=1}^{n} q_{i} \lambda^{i} d R^{i}, \quad \omega_{21}=\sum_{i=1}^{n} p_{i} d R^{i}, \quad \omega_{22}=\sum_{i=1}^{n} q_{i} d R^{i}
$$

satisfy the gl(2) structure equations,

$$
d \omega_{a b}=\sum_{c=1}^{2} \omega_{a c} \wedge \omega_{c b},
$$

$a, b=1,2$, which are equivalent to $\partial_{j} p_{i}=a_{i j} p_{i}, \partial_{j} q_{i}=a_{i j} q_{i}$.

A system satisfying either of the equivalent conditions (a), (b), or (c) is automatically semiHamiltonian.

Proof. The equivalence of (b) and (c) can be seen as follows. Considering reciprocal transformation (2), we note the relations $\partial_{i} A=\lambda^{i} \partial_{i} B, \partial_{i} M=\lambda^{i} \partial_{i} N$. Requiring that the transformed characteristic speed (3) depends on the variable $R^{i}$ only, namely, $\partial_{j} \tilde{\lambda}^{i}=0$ for every $j \neq i$, we obtain all first-order partial derivatives of $A, B, M, N$. Comparing the relations $\partial_{j} \tilde{\lambda}^{i}=\partial_{j} \tilde{\lambda}^{k}=\partial_{j} \tilde{\lambda}^{l}=0$, we obtain first-order relations (6). This allows one to set $a_{i j}=p_{j} \lambda^{i}+q_{j}$, leading to

$$
\begin{gathered}
d A=A \omega_{11}+B \omega_{12}, \quad d B=A \omega_{21}+B \omega_{22}, \\
d M=M \omega_{11}+N \omega_{12}, \quad d N=M \omega_{21}+N \omega_{22},
\end{gathered}
$$

where $\omega_{a b}$ are as in (7). The compatibility conditions of these relations are nothing but $g l(2)$ structure equations (8). A direct calculation shows that they are equivalent to $\partial_{j} p_{i}=a_{i j} p_{i}, \partial_{j} q_{i}=a_{i j} q_{i}$. By construction, in the new independent variables $\tilde{x}, \tilde{t}$, the system reduces to $n$ uncoupled Hopf equations, $R_{\tilde{t}}^{i}=f^{i}\left(R^{i}\right) R_{\tilde{x}}^{i}$, where $f^{i}$ are arbitrary functions (possibly, constants). Note that non-constant $f^{i}$ can be reduced to $R^{i}$ via a reparametrisation of Riemann invariants.

Since characteristics of a Hopf equation are straight lines, and reciprocal transformations are nothing but non-local changes of variables depending on a solution, this also establishes the implication (b) $\Longrightarrow$ (a) [equivalently, (c) $\Longrightarrow$ (a)].

As for the less elementary implication, (a) $\Longrightarrow$ (c), let us first consider the case $n=4$. Equation (5) gives $E, F, G$, and $H$ as functions of the characteristic speeds $\lambda^{i}$ and their derivatives, $\lambda_{x}^{i}=\sum_{k} \lambda_{k}^{i} R_{x}^{k}$, 
$\lambda_{t}^{i}=\sum_{k} \lambda_{k}^{i} \lambda^{k} R_{x}^{k}$ (we fix a solution so that $R^{i}$ and $\lambda^{i}$ become the functions of $x, t$ ). Substituting these expressions into (4), we obtain polynomial equations in the differential variables $R_{x}^{i}, R_{x x}^{i}, R_{x x x}^{i}$. Since the characteristic 4-web is required to be linearizable on every solution, the equations split with respect to these variables. Thus, equating to zero coefficients at $R_{x x x}^{i}$, we get first-order relations (6). Further, taking coefficients at $R_{x x}^{i} R_{x}^{j}$ and differentiating relations (6) with respect to $R^{k}$ yield all second-order relations for $\lambda^{i}$ that are equivalent to $g l(2)$ structure equations (8).

Now the general case $n>4$ readily follows. Let us fix four pairwise distinct indices $i, j, k$, and $l$, say $1,2,3$, and 4 . Consider special solutions to system (1) such that $R^{s}=$ const, $s>4$. This reduces system (1) to a 4-component system for $R^{1}, \ldots, R^{4}$, with linearizable characteristic 4 -webs. Therefore we have all relations (6), as well as all other necessary conditions involving indices 1, 2, 3, and 4 . The rest follows from the fact that every linearizability condition involves maximum 4 distinct indices.

Finally, the semi-Hamiltonian property follows from the fact that a system of uncoupled Hopf equations is automatically semi-Hamiltonian, and reciprocal transformations preserve the semi-Hamiltonian property.

Remark. Note that the equations for $A, B$ and $M, N$ uncouple and the forms $\omega_{a b}$ verify $g l(2)$ structure equations. Therefore $(A, B)$ and $(M, N)$ can be interpreted as sections of a flat 2-dimensional vector bundle over the hodograph space.

Example. The simplest example of an $n$-component system with linearizable characteristics is provided by $n$ uncoupled Euler equations,

$$
R_{t}^{i}=R^{i} R_{x}^{i}
$$

Remarkably, it appears as the modulational Whitham system for the Benjamin-Ono equation. ${ }^{7}$ Applying to this system reciprocal transformations, one obtains generic systems with linearizable characteristics. Explicitly, let us take two conservation laws,

$$
\begin{aligned}
& A d t+B d x=\left[\sum\left(\left(f^{k}\right)^{\prime} R^{k}-f^{k}\right)\right] d t+\left[\sum\left(f^{k}\right)^{\prime}\right] d x, \\
& M d t+N d x=\left[\sum\left(\left(g^{k}\right)^{\prime} R^{k}-g^{k}\right)\right] d t+\left[\sum\left(g^{k}\right)^{\prime}\right] d x,
\end{aligned}
$$

where $f^{k}\left(R^{k}\right)$ and $g^{k}\left(R^{k}\right)$ are arbitrary functions, and prime indicates derivative. The transformed characteristic speeds take the form

$$
\tilde{\lambda}^{i}=\frac{\lambda^{i} B-A}{M-\lambda^{i} N}=-\frac{\sum\left[\left(f^{k}\right)^{\prime}\left(R^{i}-R^{k}\right)+f^{k}\right]}{\sum\left[\left(g^{k}\right)^{\prime}\left(R^{i}-R^{k}\right)+g^{k}\right]} .
$$

This formula gives characteristic speeds of generic systems with linearizable characteristics. Examples of this type appeared as hydrodynamic reductions of integrable hydrodynamic chains in Ref. 18 . Degenerations can be obtained by replacing some of the Euler equations by linear equations, $R_{t}^{i}=c^{i} R_{x}^{i}$, $c^{i}=$ const . In particular, starting with $n$ linear equations and applying reciprocal transformations, one obtains

$$
\tilde{\lambda}^{i}=-\frac{\sum\left(c^{i}-c^{k}\right) f^{k}\left(R^{k}\right)}{\sum\left(c^{i}-c^{k}\right) g^{k}\left(R^{k}\right)},
$$

which is a general formula for characteristic speeds of linearly degenerate semi-Hamiltonian systems with constant cross-ratios. ${ }^{11}$ In this limiting case, the characteristic web is parallelizable on every solution.

\section{IV. $3 \times 3$ SYSTEMS WITH LINEARIZABLE CHARACTERISTICS}

Even though for $n=3$ relations (6) are vacuous, we can always represent $a_{i j}$ in the form $a_{i j}=$ $p_{j} \lambda^{i}+q_{j}$. Explicitly,

$$
p_{j}=\frac{a_{i j}-a_{k j}}{\lambda^{i}-\lambda^{k}}, \quad q_{j}=\frac{a_{k j} \lambda^{i}-a_{i j} \lambda^{k}}{\lambda^{i}-\lambda^{k}},
$$

$i, k \neq j$. Let us introduce the forms $\omega_{a b}$ by formulae (7), where the summation is from 1 to 3 . We have 


$$
\begin{gathered}
\omega_{11}=\frac{a_{31}-a_{21}}{\lambda^{3}-\lambda^{2}} \lambda^{1} d R^{1}+\frac{a_{12}-a_{32}}{\lambda^{1}-\lambda^{3}} \lambda^{2} d R^{2}+\frac{a_{23}-a_{13}}{\lambda^{2}-\lambda^{1}} \lambda^{3} d R^{3}, \\
\omega_{12}=\frac{a_{21} \lambda^{3}-a_{31} \lambda^{2}}{\lambda^{3}-\lambda^{2}} \lambda^{1} d R^{1}+\frac{a_{32} \lambda^{1}-a_{12} \lambda^{3}}{\lambda^{1}-\lambda^{3}} \lambda^{2} d R^{2}+\frac{a_{13} \lambda^{2}-a_{23} \lambda^{1}}{\lambda^{2}-\lambda^{1}} \lambda^{3} d R^{3}, \\
\omega_{21}=\frac{a_{31}-a_{21}}{\lambda^{3}-\lambda^{2}} d R^{1}+\frac{a_{12}-a_{32}}{\lambda^{1}-\lambda^{3}} d R^{2}+\frac{a_{23}-a_{13}}{\lambda^{2}-\lambda^{1}} d R^{3}, \\
\omega_{22}=\frac{a_{21} \lambda^{3}-a_{31} \lambda^{2}}{\lambda^{3}-\lambda^{2}} d R^{1}+\frac{a_{32} \lambda^{1}-a_{12} \lambda^{3}}{\lambda^{1}-\lambda^{3}} d R^{2}+\frac{a_{13} \lambda^{2}-a_{23} \lambda^{1}}{\lambda^{2}-\lambda^{1}} d R^{3} .
\end{gathered}
$$

Proposition 4. System (1) can be transformed by a reciprocal transformation to 3 uncoupled Hopf equations if and only if the forms $\omega_{a b}$ satisfy gl(2) structure equations (8).

Proof. This proposition claim can be proved exactly as in Theorem (3). Namely, requiring $\partial_{j} \tilde{\lambda}^{i}=0$ for any $j \neq i$, we obtain all first-order partial derivatives of $A, B, M$, and $N$ in the form

$$
\begin{gathered}
d A=A \omega_{11}+B \omega_{12}, \quad d B=A \omega_{21}+B \omega_{22}, \\
d M=M \omega_{11}+N \omega_{12}, \quad d N=M \omega_{21}+N \omega_{22},
\end{gathered}
$$

where $\omega_{a b}$ are as above. The compatibility conditions of these relations are the $g l(2)$ structure equations.

Due to the complexity of linearizability conditions for 3-webs, we were unable to prove the analogue of Theorem (3) for $n=3$. Thus, we can only formulate the following conjecture.

Conjecture 5. For 3-component system (1), the following conditions are equivalent:

(a) System (1) has a linearizable characteristic 3-web on every solution.

(b) System (1) can be transformed by a reciprocal transformation to 3 uncoupled Hopf equations.

(c) The forms $\omega_{a b}$ satisfy gl(2) structure equations (8).

Any system satisfying either of the above conditions is automatically semi-Hamiltonian.

Discussion. The equivalence of (b) and (c) is proved in Proposition 4.

The implication (b) $\Longrightarrow$ (a), equivalently, (c) $\Longrightarrow$ (a), is also straightforward: characteristics of a Hopf equation are straight lines, and reciprocal transformations are non-local changes of variables depending on a solution.

The main problem is the converse implication, $(\mathrm{a}) \Longrightarrow(\mathrm{c})$, which is a highly nontrivial computational challenge due to the complexity of linearizability conditions for 3-webs. This calculation seems to be out of reach for the modern computer algebra systems.

Remark. The Gronwall conjecture ${ }^{14}$ states that, modulo projective equivalence, a linearizable non-hexagonal 3-web has a unique linear representation. While this is still open at the level of individual webs (for partial results, see Refs. 1, 6, and 23), our results show that the natural analogue of this statement holds at the level of systems. Namely, if a system can be decoupled by a reciprocal transformation, then the transformation is unique up to linear changes of $x$ and $t$.

\section{CONCLUDING REMARKS}

It remains a considerable computational challenge to establish the implication $(a) \Longrightarrow(c)$ of Conjecture 5. The difference with the case $n>3$ can be explained geometrically as follows. Any 4 -subweb of a planar $n$-web defines a unique projective connection $\nabla$. The 4 -subweb is linearizable if and only if all web leaves are geodesics of $\nabla$, and the curvature of the connection vanishes. In fact, the functions $E, F, G$, and $H$ defined by Eq. (5) (where $i$ now runs over the indices of the 4 -subweb) 
are, up to constant factors, the so-called Thomas coefficients of $\nabla$ (see Ref. 17). The flatness of $\nabla$ manifests itself in Eq. (4). On the contrary, for $n=3 \mathrm{Eq}$. (5) is insufficient to define a unique projective connection.

\section{ACKNOWLEDGMENTS}

This research was supported by FAPESP Grant No. 2014/17812-0. S.I.A. thanks the Mathematical Department of Loughborough University, where this study was initiated, for a kind hospitality.

${ }^{1}$ Agafonov, S. I., "Gronwall's conjecture for 3-webs with infinitesimal symmetries," e-print arXiv:1411.0874.

${ }^{2}$ Akivis, M. A., Goldberg, V. V., and Lychagin, V. V., "Linearizability of $d$-webs, $d \geq 4$, on two-dimensional manifolds," Sel. Math. 10(4), 431-451 (2004).

${ }^{3}$ Blaschke, W. and Bol, G., Geometrie der Gewebe, Topologische Fragen der Differentialgeometrie (Springer, Berlin, 1938).

${ }^{4}$ Blaschke, W., Einführung in die Geometrie der Waben (Birkhäuser Verlag, Basel und Stuttgart, 1955), p. 108.

${ }^{5}$ Bol, G., "Topologische Fragen der Differentialgeometrie 31. Geradlinige Kurvengewebe," Abh. Math. Semin. Univ. Hamburg 8(1), 264-270 (1931).

${ }^{6}$ Bol, G., "Topologische Fragen der Differentialgeometrie 65. Ueber Geradengewebe," Ann. Math. Pura Appl. 17(1), 45-58 (1938).

${ }^{7}$ Dobrokhotov, S. Yu. and Krichever, I. M., "Multiphase solutions of the Benjamin-Ono equation and their averaging," Math. Notes 49(5-6), 583-594 (1991).

${ }^{8}$ Ferapontov, E. V., "Reciprocal transformations and their invariants," Differ. Equations 25(7), 898-905 (1989); Ferapontov, E. V., Autotransformations with respect to the solution and hydrodynamic symmetries," ibid. 27(7), 885-895 (1991).

${ }^{9}$ Ferapontov, E. V., "Systems of three differential equations of hydrodynamic type with a hexagonal 3-web of characteristics on solutions," Funct. Anal. Appl. 23(2), 151-153 (1989).

${ }^{10}$ Ferapontov, E. V., "Integration of weakly nonlinear semi-Hamiltonian systems of hydrodynamic type by the methods of web theory," Math. USSR-Sbornik 71(1), 65-79 (1992).

${ }^{11}$ Ferapontov, E. V., "Integration of weakly nonlinear hydrodynamic systems in Riemann invariants," Phys. Lett. A 158 , $112-118$ (1991).

${ }^{12}$ Goldberg, V. V. and Lychagin, V. V., "On the Blaschke conjecture for 3-webs," J. Geom. Anal. 16(1), 69-115 (2006).

${ }^{13}$ Grifone, J., Muzsnay, Z., and Saab, J., "On the linearizability of 3-webs," in Proceedings of the Third World Congress of Nonlinear Analysis, Part 4 (Catania, 2000) [Nonlinear Anal.: Theory, Methods Appl. 47(4), 2643-2654 (2001)].

${ }^{14}$ Gronwall, T. H., "Sur les équations entre trois variables représentables par les nomogrammes à points aligné," J. Liouville 8, 59-102 (1912).

${ }^{15}$ Hénaut, A., "Sur la linéarisation des tissus de $\mathbb{C}^{2}$," Topology 32(3), 531-542 (1993).

${ }^{16}$ Liu, T. P., "Development of singularities in the nonlinear waves for quasi-linear hyperbolic PDEs," J. Differ. Equations $\mathbf{3 3}$, 92-111 (1979).

${ }^{17}$ Pirio, L., "Sur la linéarisation des tissus," L'Enseign. Math. 55(3-4), 285-328 (2009).

18 Pavlov, M. V., "Integrable hydrodynamic chains," in Nonlinear Physics: Theory and Experiment, II (Gallipoli, 2002) (World Scientific Publishing, River Edge, NJ, 2003), p. 101-107.

${ }^{19}$ Rozdestvenskii, B. L. and Sidorenko, A. D., "On the impossibility of 'gradient catastrophe' for weakly nonlinear systems," Z. Vycisl. Mat. Mat. Fiz. 7, 1176-1179 (1967).

${ }^{20}$ Sasaki, T., Projective Differential Geometry and Linear Homogeneous Differential Equations, Rokko Lectures in Mathematics (Kobe University, 1999), Vol. 5.

${ }^{21}$ Serre, D., Systems of Conservation Laws 1: Hyperbolicity, Entropies, Shock Waves (Cambridge University Press, 1999), p. 263; Systems of Conservation Laws 2: Geometric Structures, Oscillations, and Initial-Boundary Value Problems (Cambridge University Press, 2000), p. 269.

${ }^{22}$ Tsarev, S. P., "Poisson brackets and one-dimensional Hamiltonian systems of hydrodynamic type," Soviet Math. Dokl. 31, 488-491 (1985). "The geometry of Hamiltonian systems of hydrodynamic type. The generalized hodograph method," Math. USSR Izvestiya 37, 397-419 (1991).

${ }^{23}$ Wang, J. S., “On the Gronwall conjecture,” J. Geom. Anal. 22(1), 38-73 (2012). 